\title{
Benchmark Problems for the Numerical Discretization of the Cahn-Hilliard Equation with a Source Term
}

\author{
Sungha Yoon, ${ }^{1}$ Hyun Geun Lee, ${ }^{2}$ Yibao Li, ${ }^{3}$ Chaeyoung Lee, ${ }^{1}$ Jintae Park, ${ }^{1}$ Sangkwon Kim, \\ Hyundong Kim, ${ }^{1}$ and Junseok Kim (iD ${ }^{1}$
}

${ }^{1}$ Department of Mathematics, Korea University, Seoul 02 841, Republic of Korea

${ }^{2}$ Department of Mathematics, Kwangwoon University, Seoul 01 897, Republic of Korea

${ }^{3}$ School of Mathematics and Statistics, Xi'an Jiaotong University, Xi'an 710 049, China

Correspondence should be addressed to Junseok Kim; cfdkim@korea.ac.kr

Received 24 September 2021; Accepted 20 November 2021; Published 6 December 2021

Academic Editor: Nikos I. Karachalios

Copyright (c) 2021 Sungha Yoon et al. This is an open access article distributed under the Creative Commons Attribution License, which permits unrestricted use, distribution, and reproduction in any medium, provided the original work is properly cited.

In this paper, we present benchmark problems for the numerical discretization of the Cahn-Hilliard equation with a source term. If the source term includes an isotropic growth term, then initially circular and spherical shapes should grow with their original shapes. However, there is numerical anisotropic error and this error results in anisotropic evolutions. Therefore, it is essential to use isotropic space discretization in the simulation of growth phenomenon such as tumor growth. To test numerical discretization, we present two benchmark problems: one is the growth of a disk or a sphere and the other is the growth of a rotated ellipse or a rotated ellipsoid. The computational results show that the standard discrete Laplace operator has severe grid orientation dependence. However, the isotropic discrete Laplace operator generates good results.

\section{Introduction}

We consider the Cahn-Hilliard $(\mathrm{CH})$ equation with the reaction term:

$$
\begin{aligned}
\frac{\partial \phi(\mathbf{x}, t)}{\partial t} & =\Delta \mu(\mathbf{x}, t)+\gamma(\phi(\mathbf{x}, t)), \quad \mathbf{x} \in \Omega, t>0, \\
\mu(\mathbf{x}, t) & =F^{\prime}(\phi(\mathbf{x}, t))-\epsilon^{2} \Delta \phi(\mathbf{x}, t),
\end{aligned}
$$

where $\phi(\mathbf{x}, t)$ is the order parameter in the domain $\Omega \subset \mathbb{R}^{d}$, $d=2,3, F(\phi)=0.25\left(\phi^{2}-1\right)^{2}$, and $\epsilon$ is a constant. The reaction term $\gamma(\phi)$ is specified in Section 3. In this paper, we present two benchmark problems for the numerical discretization of the $\mathrm{CH}$ equation with a source term in $2 \mathrm{D}$ and $3 \mathrm{D}$.

The $\mathrm{CH}$ equation without a source term was derived for minimizing the interface in binary alloys $[1,2]$. This topic has been studied in various fields such as multiphase flows [3-5], image inpainting [6], phase-field model [7-10], and microstructures with elastic inhomogeneity [11]. Since the common behavior of the $\mathrm{CH}$ equation without source terms is minimizing the interface, the anisotropic space discretization error is not noticeable. However, in the case of a growth model, anisotropic discretization may have critical problems. In particular, tumor growth simulation with the $\mathrm{CH}$ equation is one of the cases. Several research studies have been conducted numerically by using the Galerkin finite element method [12-15]. Fakih [16, 17] analyzed the asymptotic behavior of the $\mathrm{CH}$ equation with source in both Dirichlet and Neumann boundary conditions by using the P1 element method. Khain and Sander [18] studied the logistic growth model of the $\mathrm{CH}$ equation. In recent years, many studies for tumor growth simulation using the finite difference method have been actively conducted [19-23].

However, the conventional space discretization scheme has a high probability of having grid-related artifact because it has anisotropic properties. Therefore, we need to use the isotropic discretization. For instance, Kumar introduced the isotropic finite difference method [24]. We use the method proposed by Kumar in this paper. The isotropic finite difference scheme was derived for symmetric dendritic solidification and reduces an observable computational bias of the numerical anisotropy. In [25], the authors introduced 
different stencils for the Laplacian, bi-Laplacian, and gradient Laplacian operators and emphasized the advantages of using the isotropic stencils. Assadi [26] applied the isotropic scheme to the Allen-Cahn-type equation.

The main purpose of this work is to present benchmark problems for the numerical discretization of the $\mathrm{CH}$ equation with a source term.

The rest of this article is organized as follows. In Section 2 , we describe two discretization schemes of the $\mathrm{CH}$ equation with a source term. We compare two schemes on several numerical experiments in Section 3. In Section 4, we provide a summary and present our conclusions.

\section{Discretization Schemes}

We consider the two discretization schemes of the $\mathrm{CH}$ equation with a source term. To solve the $\mathrm{CH}$ equation numerically, we first define a $2 \mathrm{D}$ discrete domain for $\Omega=(a, b) \times(c, d)$, i.e., $\Omega_{h}=\left\{\left(x_{i}, y_{j}\right): x_{i}=a+(i-0.5) h, y_{j}\right.$ $\left.=c+(j-0.5) h, 1 \leq i \leq N_{x}, 1 \leq j \leq N_{y}\right\}$, where $N_{x}$ and $N_{y}$ are positive even integers, and $h=(b-a) / N_{x}=(d-c) / N_{y}$ is the grid size. Let $\phi_{i j}^{n}$ and $\mu_{i j}^{n}$ be approximations of $\phi\left(x_{i}, y_{j}, t_{n}\right)$ and $\mu\left(x_{i}, y_{j}, t_{n}\right)$, respectively, where $t_{n}=n \Delta t$ and $\Delta t$ is the time step. We use the periodic boundary condition for $\phi$ and $\mu$ as follows:

$$
\begin{aligned}
\phi_{i, 0} & =\phi_{i, N_{y},}, \\
\phi_{i, N_{y}+1} & =\phi_{i, 1}, \\
\phi_{0, j} & =\phi_{N x, j}, \\
\phi_{N x+1, j} & =\phi_{1, j},
\end{aligned}
$$

$$
\begin{aligned}
\mu_{i, 0} & =\mu_{i, N_{y}}, \\
\mu_{i, N_{y}+1} & =\mu_{i, 1}, \\
\mu_{0, j} & =\mu_{N x, j}, \\
\mu_{N_{x}+1, j} & =\mu_{1, j} .
\end{aligned}
$$

For simplicity, we take Eyre's nonlinearly stabilized splitting scheme [27]. The first scheme is the following with the standard discrete Laplace operator:

$$
\begin{aligned}
\frac{\phi_{i j}^{n+1}-\phi_{i j}^{n}}{\Delta t} & =\Delta_{S} \mu_{i j}^{n+1}+\gamma\left(\phi_{i j}^{n}\right), \\
\mu_{i j}^{n+1} & =\left(\phi_{i j}^{n+1}\right)^{3}-\phi_{i j}^{n}-\epsilon^{2} \Delta_{S} \phi_{i j}^{n+1},
\end{aligned}
$$

where the standard discrete Laplace operator $\Delta_{S}$ is defined as

$$
\Delta_{S} \phi_{i j}=\frac{\left(\phi_{i-1, j}+\phi_{i+1, j}+\phi_{i, j-1}+\phi_{i, j+1}-4 \phi_{i j}\right)}{h^{2}} .
$$

The second scheme is the isotropic discretization of the Laplace operator [24]:

$$
\begin{aligned}
\frac{\phi_{i j}^{n+1}-\phi_{i j}^{n}}{\Delta t} & =\Delta_{I} \mu_{i j}^{n+1}+\gamma\left(\phi_{i j}^{n}\right), \\
\mu_{i j}^{n+1} & =\left(\phi_{i j}^{n+1}\right)^{3}-\phi_{i j}^{n}-\epsilon^{2} \Delta_{I} \phi_{i j}^{n+1},
\end{aligned}
$$

where the isotropic discrete Laplace operator $\Delta_{I}$ is defined as

$$
\Delta_{I} \phi_{i j}=\frac{\left[4\left(\phi_{i+1, j}+\phi_{i-1, j}+\phi_{i, j+1}+\phi_{i, j-1}\right)+\phi_{i+1, j+1}+\phi_{i-1, j+1}+\phi_{i-1, j-1}+\phi_{i+1, j-1}-20 \phi_{i j}\right]}{\left(6 h^{2}\right)} .
$$

Similarly, we can define both discrete Laplace operators in a 3D discrete domain $\Omega_{h}=\left\{\left(x_{i}, y_{j}, z_{k}\right): x_{i}=a+(i-0.5)\right.$ $h, y_{j}=c+(j-0.5) h, z_{k}=e+(k-0.5) h, 1 \leq i \leq N_{x}, 1 \leq$ $\left.j \leq N_{y}, 1 \leq k \leq N_{z}\right\}$ for $\Omega=(a, b) \times(c, d) \times(e, f)$, where
$N_{x}, N_{y}$, and $N_{z}$ are positive even integers, and $h=(b-$ a) $/ N_{x}=(d-c) / N_{y}=(f-e) / N_{z}$ is the grid size. The standard discrete Laplace operator is defined as

$$
\Delta_{S} \phi_{i j k}=\frac{\left(\phi_{i-1, j, k}+\phi_{i+1, j, k}+\phi_{i, j-1, k}+\phi_{i, j+1, k}+\phi_{i, j, k-1}+\phi_{i, j, k+1}-6 \phi_{i j k}\right)}{h^{2}} .
$$

The isotropic discrete Laplace operator is defined as

$$
\begin{aligned}
\Delta_{I} \phi_{i j k}= & {\left[20\left(\phi_{i-1, j, k}+\phi_{i+1, j, k}+\phi_{i, j-1, k}+\phi_{i, j+1, k}+\phi_{i, j, k-1}+\phi_{i, j, k+1}\right)\right.} \\
& +6\left(\phi_{i-1, j-1, k}+\phi_{i-1, j+1, k}+\phi_{i+1, j-1, k}+\phi_{i+1, j+1, k}+\phi_{i, j-1, k-1}\right. \\
& +\phi_{i, j-1, k+1}+\phi_{i, j+1, k-1}+\phi_{i, j+1, k+1}+\phi_{i-1, j, k-1}+\phi_{i-1, j, k+1}
\end{aligned}
$$




$$
\begin{aligned}
& \left.+\phi_{i+1, j, k-1}+\phi_{i+1, j, k+1}\right)+\phi_{i-1, j-1, k-1}+\phi_{i-1, j-1, k+1} \\
& +\phi_{i-1, j+1, k-1}+\phi_{i-1, j+1, k+1}+\phi_{i+1, j-1, k-1}+\phi_{i+1, j-1, k+1} \\
& \left.+\phi_{i+1, j+1, k-1}+\phi_{i+1, j+1, k+1}-200 \phi_{i j k}\right] /\left(48 h^{2}\right)^{-1} .
\end{aligned}
$$

We use the nonlinear multigrid method [28-30] to solve the above discrete $\mathrm{CH}$ equations.

\section{Numerical Results and Discussion}

3.1. Convergence Test. We start with numerical convergence tests of two discrete Laplace schemes. We consider the 2D heat equation with the periodic boundary condition (3) on a domain $\Omega=(0,1) \times(0,1)$ :

$$
u_{t}(x, y, t)=\Delta u(x, y, t), \quad \text { for }(x, y) \in \Omega, t>0 .
$$

For example, we use the following initial condition for equation (9):

$$
u(x, y, 0)=\cos (2 \pi x) \cos (2 \pi y)
$$

Then, the exact solution is $u(x, y, t)=e^{-8} \pi^{2} t \cos$ $(2 \pi x) \cos (2 \pi y)$. Numerical convergence tests of two different schemes, standard and isotropic schemes, are performed with $N_{x}=N_{y}=2^{n}$ and $h=1 / N_{x}, \Delta t=10^{-9}$. The final time is $T=N_{t} \Delta t$ for $n=3,4,5$, and 6. Here, $N_{t}=20$ is used. Let $e_{i j}^{h}$ : $=u_{i j}^{N_{t}}-u\left(x_{i}, y_{j}, T\right)$ be the numerical error in $\Omega_{h}$. We define the discrete $l_{2}$-norm of the error as $\|e\|_{2}=\sqrt{\sum_{i=1}^{N_{x}} \sum_{j=1}^{N_{y}} e_{i j}^{2} /\left(N_{x} N_{y}\right)}$. Then, the rate of convergence is $\log _{2}\left(\left\|e^{h}\right\|_{2} /\left\|e^{h / 2}\right\|_{2}\right)$. Table 1 shows the discrete $l_{2}$-norm of errors and the rates of convergence with the two different formulas. Numerical experimentation suggests that both schemes are second-order accurate in space.

3.2. Comparison of the Two Schemes for an Isotropic Initial Shape. We consider the tumor growth simulation parameters in [12]:

$$
\gamma(\phi)=\lambda_{g}(1+\phi)^{2}(1-\phi)^{2}-\frac{\lambda_{d}}{2}(1+\phi),
$$

where $\lambda_{g}$ and $\lambda_{d}$ are the growth and death coefficients, respectively. We use $\lambda_{g}=280$ and $\lambda_{d}=92$.

Figure 1 shows a plot of $\gamma(\phi)=280(1+\phi)^{2}(1-\phi)^{2}$ $-46(1+\phi)$. Note that $\gamma(\phi)=0$ when $\phi=-1$ and $\phi \approx-0.957109,0.688032,1.26908$.

3.2.1. 2D Case. We present the evolution of a disk by using the two types of schemes: standard and isotropic. The initial condition in the domain $\Omega=(-0.6,0.6) \times(-0.6,0.6)$ is given by

$$
\phi(x, y, 0)=\tanh \left(\frac{0.1-\sqrt{x^{2}+y^{2}}}{\sqrt{2} \epsilon}\right) .
$$

In this test, the uniform space step size $h=1.2 / 128$, the time step size $\Delta t=10 h^{2}$, and $\epsilon=0.0125$ are used. Figure 2 shows the circular evolution of the disk with standard and isotropic schemes, respectively, up to the final time $t=1200 \Delta t$ at every $120 \Delta t$.

There is a significant computational bias for diagonal directions in the case with the standard scheme. On the other hand, the computational bias in the case with the isotropic scheme seems not large. In order to compare both schemes in more detail, we compare the ratios such as

$$
r_{d}=\frac{l^{2}}{4 \pi A}
$$

where $l$ implies the perimeter of zero-level contour and $A$ implies the area enclosed by the contour. Note that $r_{d} \approx 1$ if an evolution maintains the form of the disk. Figure 3 shows the ratios $r_{d}$ of the standard scheme and the isotropic scheme.

Thus, we can confirm that an initially circular shape actually evolves closer to the original shape with the isotropic scheme while the standard scheme does not.

3.2.2. $3 D$ Case. We further present the evolution of sphere in $\Omega=(-0.6,0.6)^{3}$ by using both standard and isotropic schemes with the initial condition given by

$$
\phi(x, y, z, 0)=\tanh \left(\frac{0.1-\sqrt{x^{2}+y^{2}+z^{2}}}{\sqrt{2} \epsilon}\right) .
$$

Figure 4 shows isosurfaces $\phi=0$ of the evolution of equation (6) with both schemes. Here, we use the uniform step size $h=1.2 / 128$, the time step $\Delta t=10 h^{2}, \epsilon=0.0125$, and the final time $t=1600 \Delta t$.

Obviously, as shown in Figure 4(b), it was tested using the isotropic scheme that grew closer to the shape of the initial condition. For the next step, we use the ratios $r_{s}$ such as each surface area over volume with appropriate scaling to compare evolutionary detail of both schemes, and hence $r_{s}$ is defined as

$$
r_{s}=\frac{s^{3}}{36 \pi V^{2}}
$$

where $S$ is a surface area of sphere and $V$ is a volume of sphere. Note that $r_{s} \approx 1$ if an evolution maintains the form of the sphere. Figure 5 shows the ratios $r_{s}$ of both schemes. Thus, we conclude that the isotropic scheme is relatively unbiased and has a correct evolution.

3.3. Comparison of the Two Schemes for an Anisotropic Initial Shape. In Section 3.2, we have performed evolutions of a sphere by using standard and isotropic schemes. In order to 
TABLE 1: $l_{2}$-norm of the errors and convergence rates of standard and isotropic schemes.

\begin{tabular}{|c|c|c|c|c|}
\hline \multirow[b]{2}{*}{$h$} & \multicolumn{2}{|c|}{ Standard } & \multicolumn{2}{|c|}{ Isotropic } \\
\hline & $l_{2}$ error & Order & $l_{2}$ error & Order \\
\hline $1 / 8$ & $3.976 \times 10^{-8}$ & & $7.636 \times 10^{-8}$ & \\
\hline $1 / 16$ & $1.009 \times 10^{-8}$ & 1.978 & $1.998 \times 10^{-8}$ & 1.934 \\
\hline $1 / 32$ & $2.533 \times 10^{-9}$ & 1.994 & $5.054 \times 10^{-9}$ & 1.983 \\
\hline $1 / 64$ & $6.340 \times 10^{-10}$ & 1.999 & $1.267 \times 10^{-9}$ & 1.996 \\
\hline
\end{tabular}

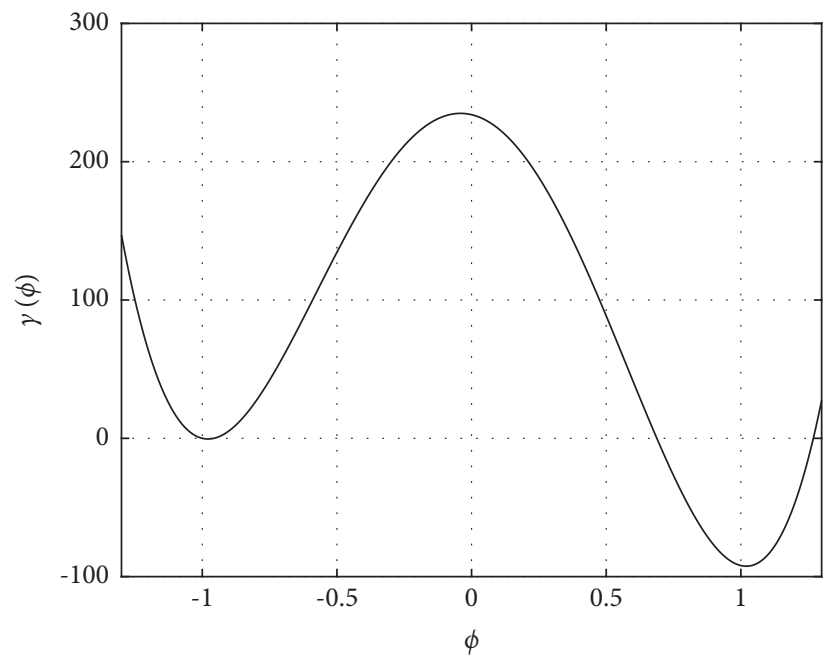

Figure 1: Plot of $\gamma(\phi)=280(1+\phi)^{2}(1-\phi)^{2}-46(1+\phi)$.

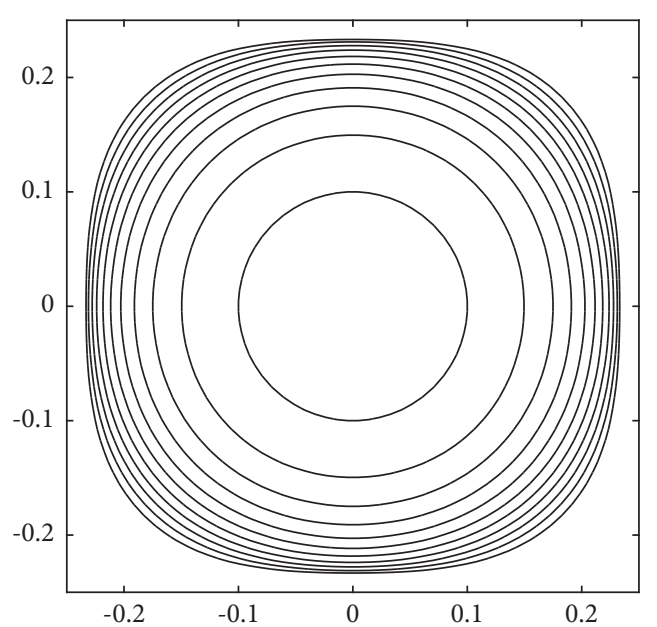

(a)

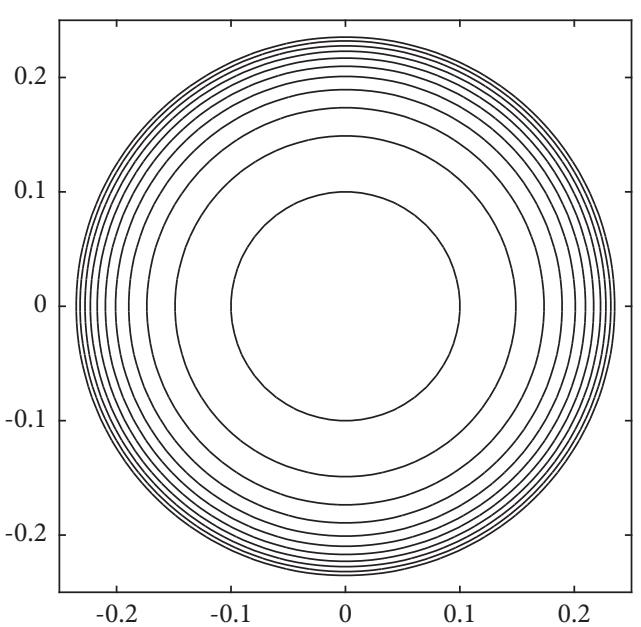

(b)

Figure 2: (a, b) Zero-level contours for the circular evolution in equation (12) with standard and isotropic schemes up to $t=1200 \Delta t$ at every $120 \Delta t$, respectively.

get a more detailed look at the grid effect, we now perform a benchmark test on an anisotropic initial shape.

3.3.1. 2D Case. We investigate tumor growth of an elliptical shape in $2 \mathrm{D}$ with $0^{\circ}$ and $45^{\circ}$ rotations in clockwise direction in $\Omega=(-2.4,2.4) \times(-2.4,2.4)$, where initial conditions are given, respectively, as follows:

$$
\begin{aligned}
& \phi(x, y, 0)=\tanh \left(\frac{0.1-\sqrt{2 x^{2}+0.25 y^{2}}}{\sqrt{2} \epsilon}\right), \\
& \phi(x, y, 0)=\tanh \left(\frac{0.1-\sqrt{2 X^{2}+0.25 Y^{2}}}{\sqrt{2} \epsilon}\right),
\end{aligned}
$$

where $X$ and $Y$ are defined as 


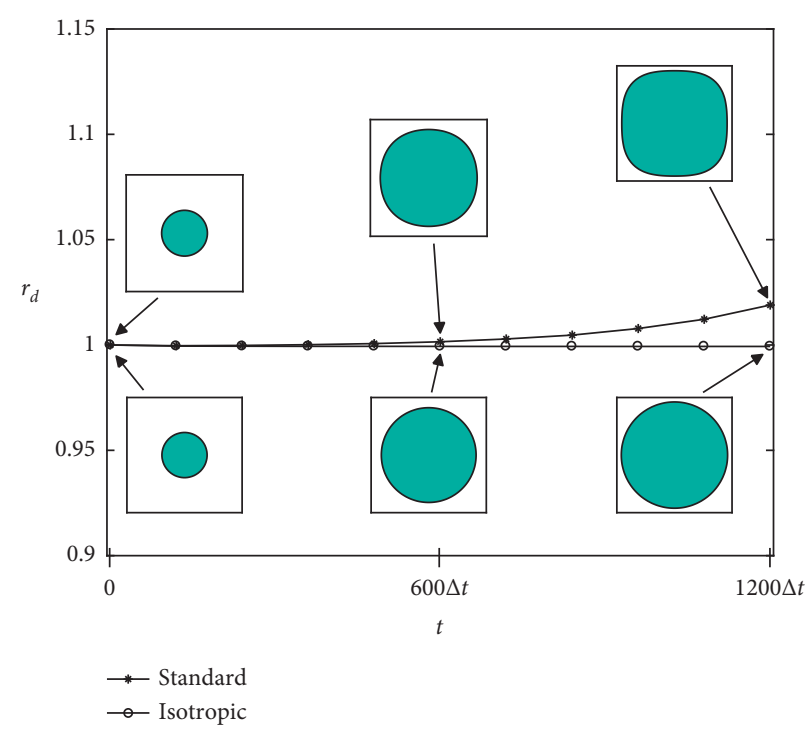

FIgURE 3: Plot of $r_{d}$ among standard and isotropic schemes.

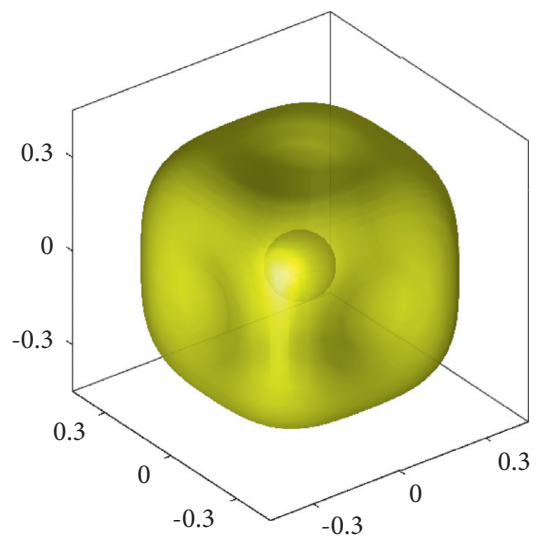

(a)

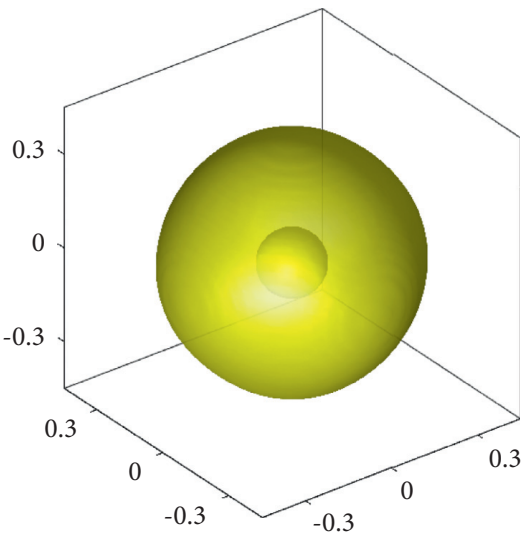

(b)

FIgURe 4: (a) Overlapped isosurfaces $\phi=0$ for evolution in equation (14) with the standard scheme at $t=0$ and $t=1600 \Delta t$. (b) Overlapped isosurfaces $\phi=0$ for evolution in equation (14) with the isotropic scheme at $t=0$ and $t=1600 \Delta t$.

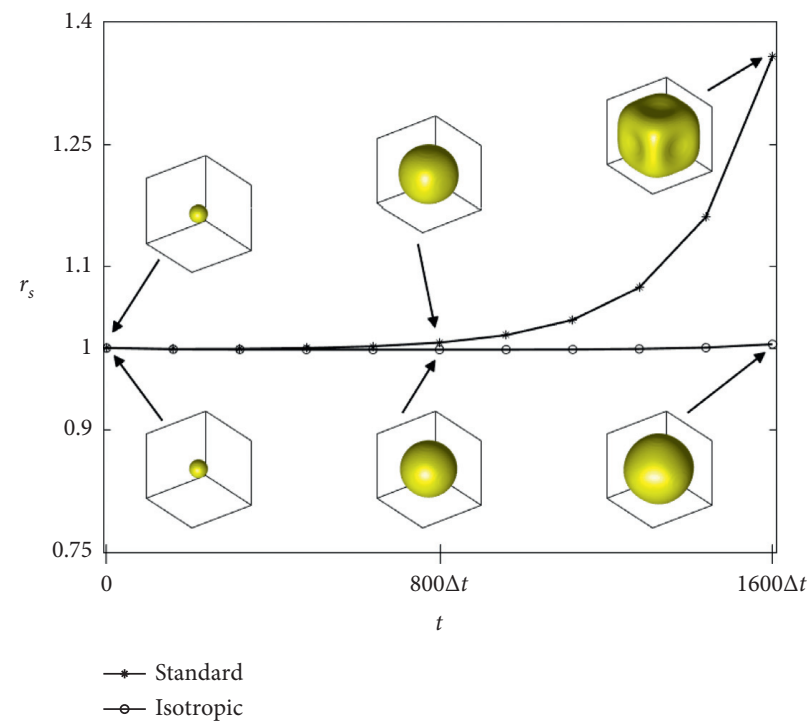

FIgURE 5: Plot of $r_{s}$ from standard and isotropic schemes. 


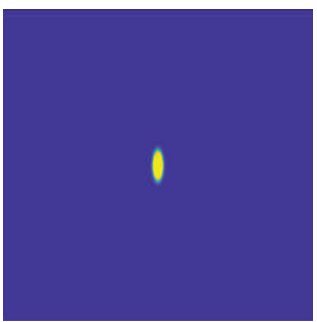

(a)

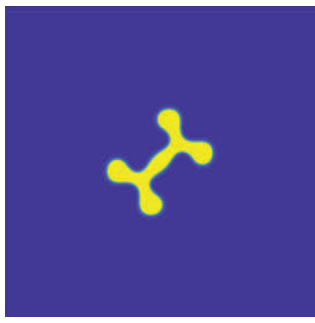

(f)

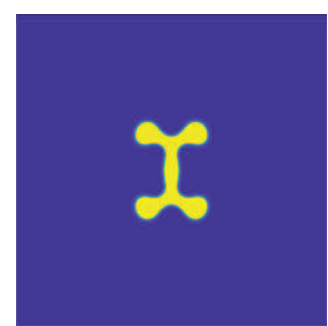

(b)

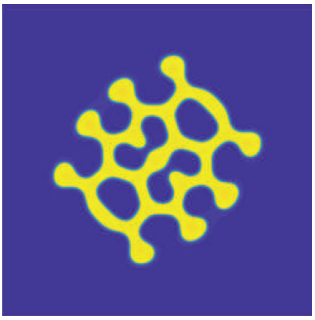

g)

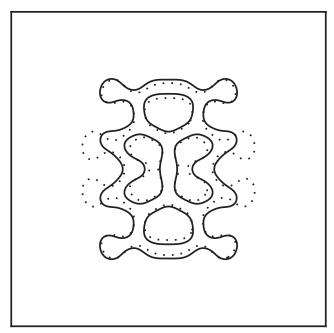

… $45^{\circ}$

$-0^{\circ}$

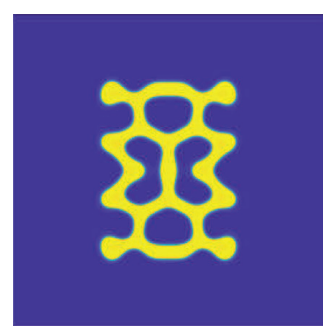

(c)

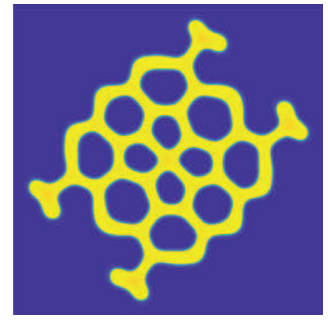

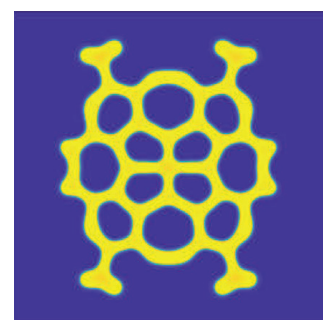

(d)

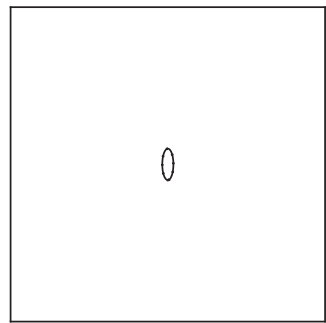

$\cdots$
$\cdots$
$-0^{\circ}$

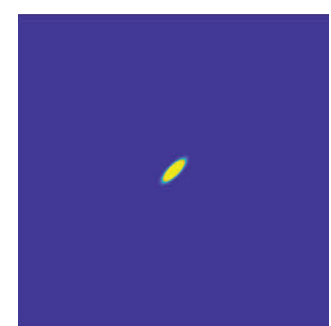

(e)

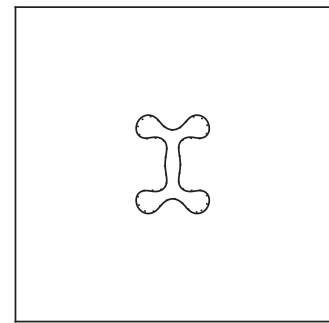

$45^{\circ}$

(h)

(i)

(j)

FIGURE 6: (a-d) Snapshots of zero-level contours for evolution in (16) using the standard scheme at $t=0,15000 \Delta t, 35000 \Delta t$, and 50000 $\Delta t$, respectively. (e-h) Snapshots of zero-level contours for evolution in (17) using the standard scheme at $t=0,15000 \Delta t, 35000 \Delta t$, and $50000 \Delta t$, respectively. (i-1) Overlapped zero-level contours for each column at $t=0,15000 \Delta t, 35000 \Delta t$, and 50000 $\Delta t$, respectively.

$$
\left(\begin{array}{l}
X \\
Y
\end{array}\right)=\left(\begin{array}{cc}
\cos \theta & \sin \theta \\
-\sin \theta & \cos \theta
\end{array}\right)\left(\begin{array}{l}
x \\
y
\end{array}\right) .
$$

Here, $\theta=-\pi / 4, h=4.8 / 512, \Delta t=h^{2}$, and $\epsilon=0.0125$ are used. We show four stages of tumor growth: $t=0,15000 \Delta t$, $35000 \Delta t$, and $50000 \Delta t$. We can see a difference of tumor growth in two kinds of space discretization. Figure 6 shows evolutions of ellipse with the standard scheme. Snapshots of zero-level contours for evolution over time in equation (16) are listed in the first row of Figure 6, and those in equation (17) are in the second row of Figure 6 . The final row shows overlapped contours for each column at $t=0$, $15000 \Delta t, 35000 \Delta t$, and $50000 \Delta t$. The results under the same conditions by using the isotropic scheme are given in Figure 7.

3.3.2. 3D Case. We further investigate the evolution of ellipsoidal shape in $\Omega=(-1.0,1.0)^{3}$, where initial conditions are given, respectively, as follows:

$$
\begin{aligned}
& \phi(x, y, z, 0)=\tanh \left(\frac{0.2-\sqrt{0.5 x^{2}+2 y^{2}+2 z^{2}}}{\sqrt{2} \epsilon}\right), \\
& \phi(x, y, z, 0)=\tanh \left(\frac{0.2-\sqrt{0.5 X^{2}+2 Y^{2}+2 Z^{2}}}{\sqrt{2} \epsilon}\right),
\end{aligned}
$$

where $X, Y$, and $Z$ are defined as

$$
\left(\begin{array}{c}
X \\
Y \\
Z
\end{array}\right)=R_{x} R_{y} R_{z}\left(\begin{array}{l}
x \\
y \\
z
\end{array}\right) .
$$

Note that $R_{x}, R_{y}$, and $R_{z}$ are rotation matrices as follows:

$$
R_{x}=\left(\begin{array}{ccc}
1 & 0 & 0 \\
0 & \cos \theta & \sin \theta \\
0 & -\sin \theta & \cos \theta
\end{array}\right),
$$




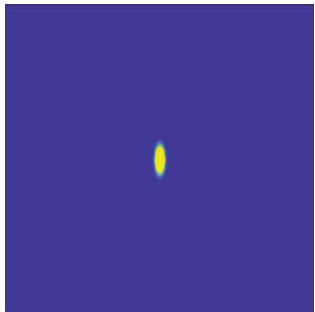

(a)

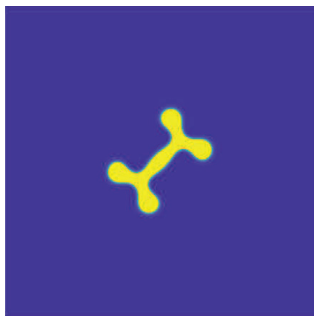

(f)

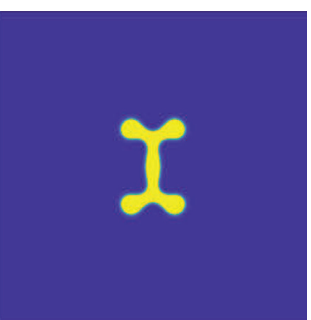

(b)

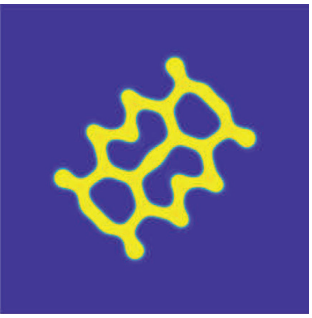

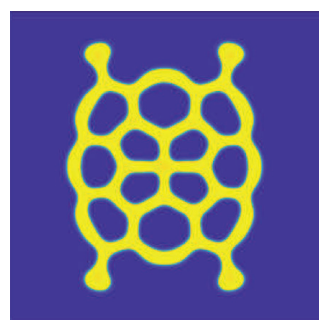

(d)
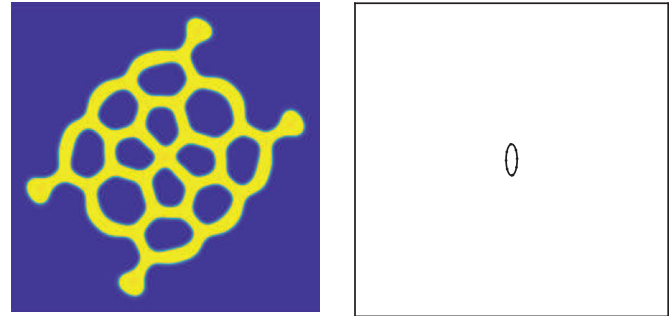

..... $45^{\circ}$

$-0^{\circ}$

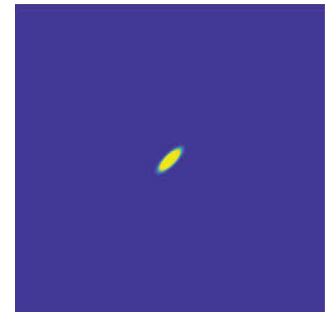

(e)

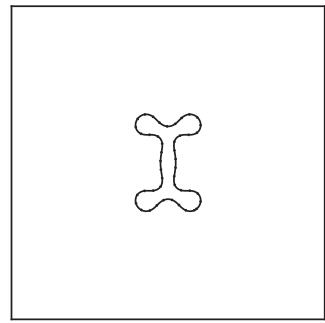

..... $45^{\circ}$ (g)

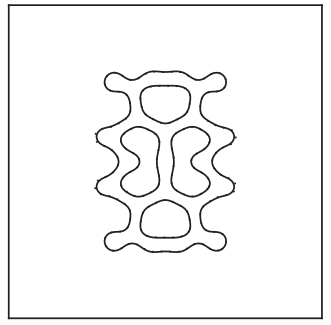

$\cdots$
$-0^{\circ}$ (h)

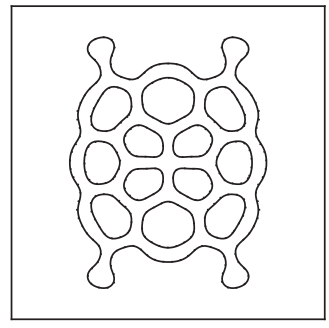

$-0^{\circ}$

(k)

(1)

Figure 7: (a-d) Snapshots of zero-level contours for evolution in equation (16) using the isotropic scheme at $t=0,15000 \Delta t$, 35000 $\Delta t$, and $50000 \Delta t$, respectively. (e-h) Snapshots of zero-level contours for evolution in equation (17) using the isotropic scheme at $t=0,15000 \Delta t$, $35000 \Delta t$, and $50000 \Delta t$, respectively. (i-l) Overlapped zero-level contours for each column at $t=0,15000 \Delta t, 35000 \Delta t$, and $50000 \Delta t$, respectively.

$$
\begin{aligned}
& R_{y}=\left(\begin{array}{ccc}
\cos \theta & 0 & -\sin \theta \\
0 & 1 & 0 \\
\sin \theta & 0 & \cos \theta
\end{array}\right), \\
& R_{z}=\left(\begin{array}{ccc}
\cos \theta & \sin \theta & 0 \\
-\sin \theta & \cos \theta & 0 \\
0 & 0 & 1
\end{array}\right),
\end{aligned}
$$

where $\theta=-\pi / 4$ and rotations are based on $x$-, $y$-, and $z$-axes, respectively. We use the uniform step size $h=2.0 / 128$, the time step size $\Delta t=10 h^{2}, \epsilon=0.0125$, and the final time $1000 \Delta t$. Figure 8 shows evolutions of ellipsoid over time $t=0,600 \Delta t$, and $1000 \Delta t$ with the standard scheme. Snapshots of isosurface $\phi=0$ for evolution over time in equation (9) are listed in the first row of Figure 8, and those in equation (10) are listed in the second row of Figure 8. The final row shows overlapped isosurfaces $\phi=0$ for each column at $t=0,600 \Delta t$, and $1000 \Delta t$, respectively. Note that we use rotation matrices to align the axes of $\theta=$ $-\pi / 4$ case with those of $\theta=0$, so we could overlap isosurfaces on identical axes. The results under the same conditions by using the isotropic scheme are listed in Figure 9. Unlike the isotropic scheme, there is a computational bias in the standard scheme. This is the reason why we should use the isotropic scheme in tumor growth simulation. 


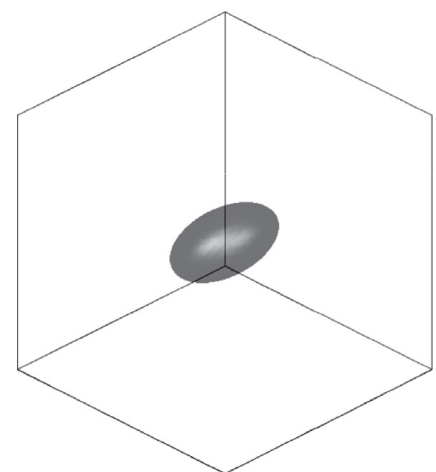

(a)

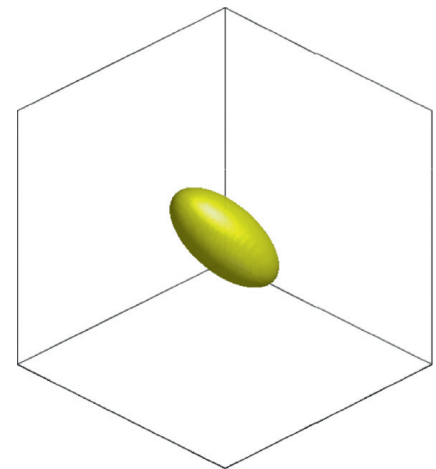

(d)

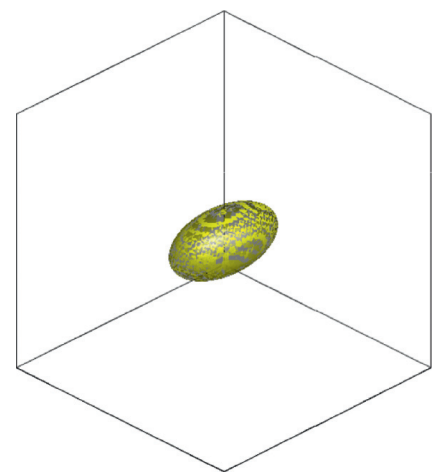

$\theta=0$
$\theta=-\pi / 4$

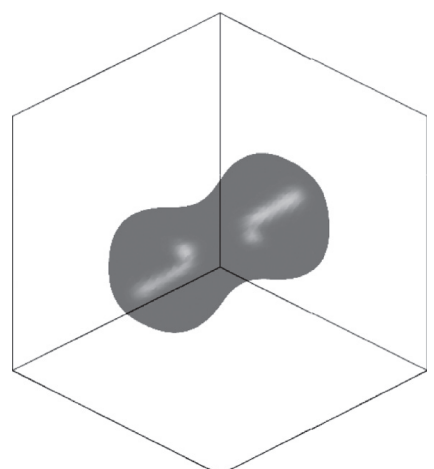

(b)

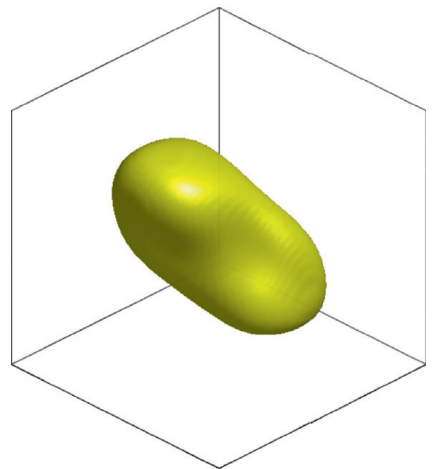

(e)

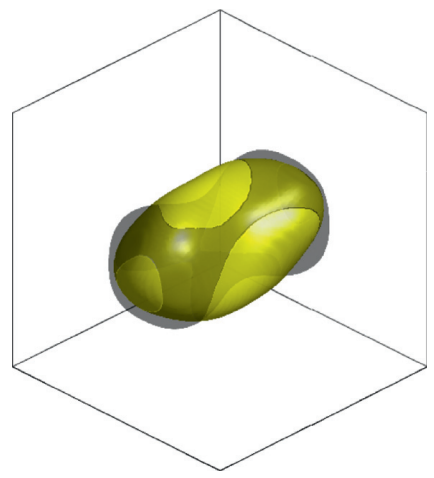

$\theta=0$
$\theta=-\pi / 4$

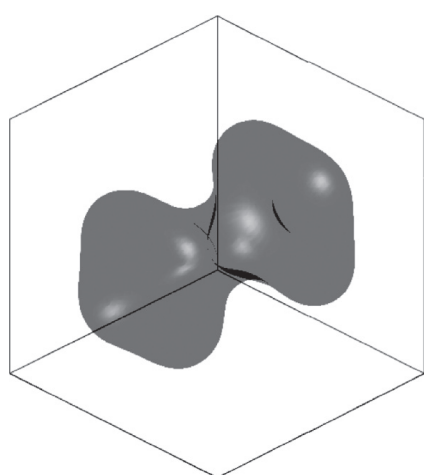

(c)

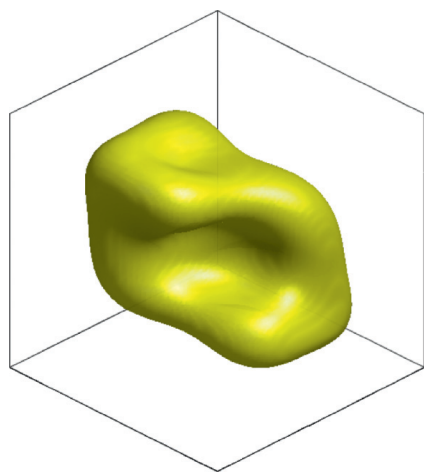

(f)

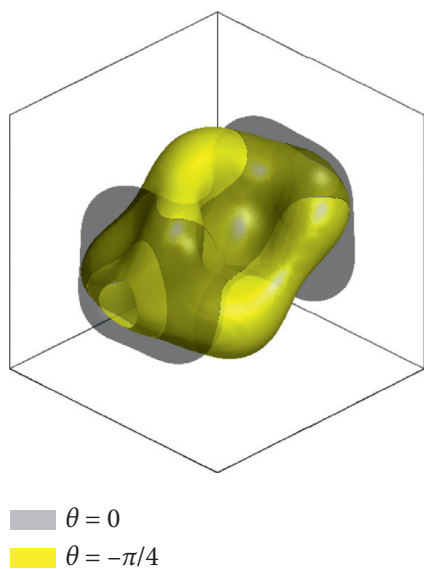

(i)

Figure 8: $(\mathrm{a}-\mathrm{c})$ Snapshots of isosurface $\phi=0$ for evolution in equation (9) using the standard scheme at $t=0,600 \Delta t$, and $1000 \Delta t$, respectively. (d-f) Snapshots of isosurface $\phi=0$ for evolution in equation (10) using the standard scheme at $t=0,600 \Delta t$, and $1000 \Delta t$, respectively. (g-i) Overlapped isosurfaces $\phi=0$ for each column at $t=0,600 \Delta t$, and $1000 \Delta t$, respectively. Note that the axes are limited to show only $(-0.6,0.6)^{3}$. 


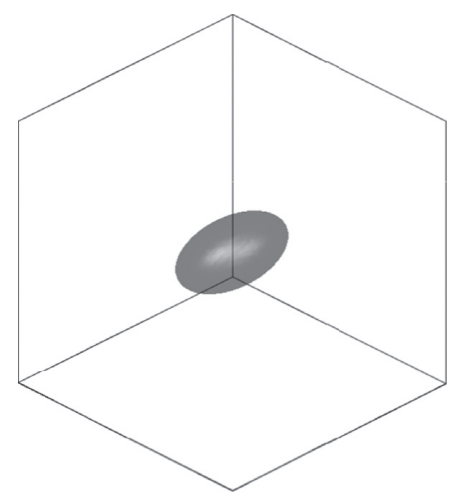

(a)

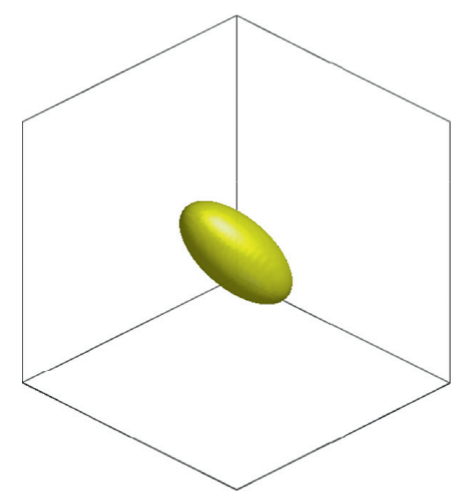

(d)

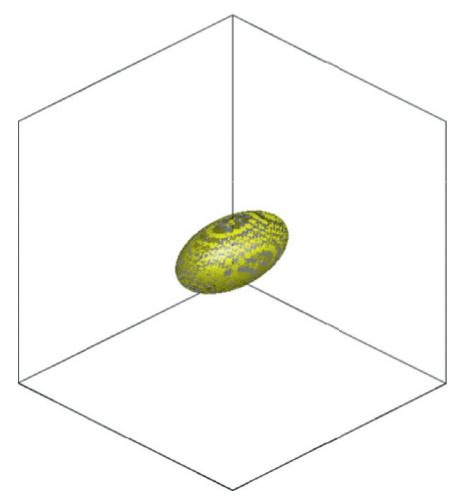

$$
\theta=0
$$$$
\theta=-\pi / 4
$$

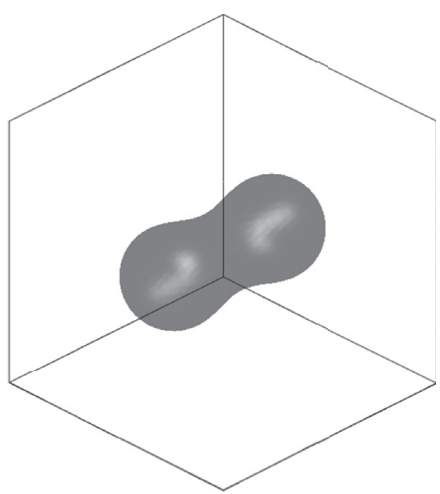

(b)

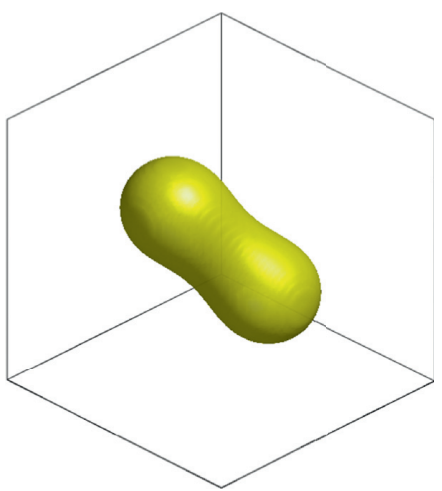

(e)

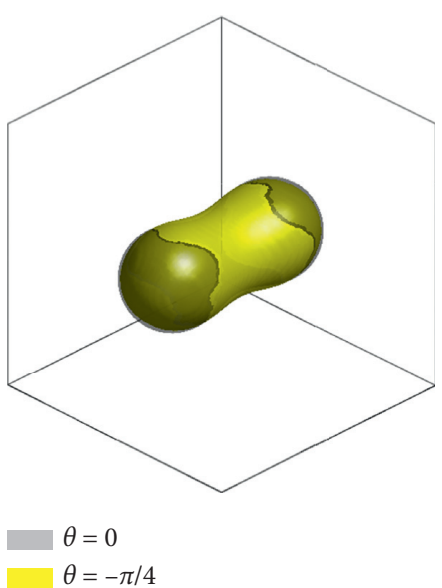

(h)

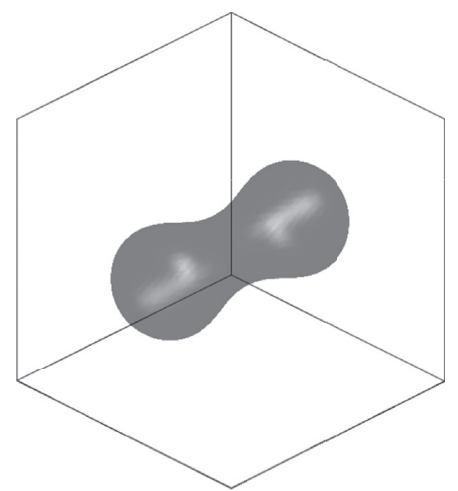

(c)

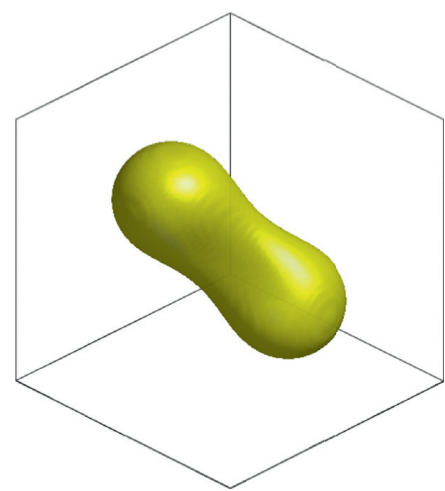

(f)

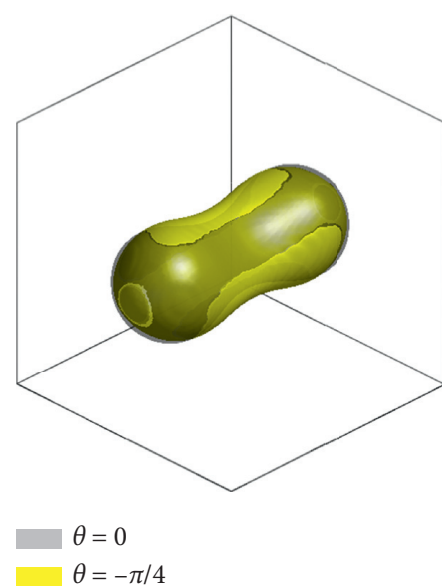

(i)

Figure 9: $(\mathrm{a}-\mathrm{c})$ Snapshots of isosurface $\phi=0$ for evolution in equation (9) using the isotropic scheme at $t=0,600 \Delta t$, and $1000 \Delta t$, respectively. ( $\mathrm{d}-\mathrm{f}$ ) Snapshots of isosurface $\phi=0$ for evolution in equation (10) using the isotropic scheme at $t=0$, 600 $\Delta t$, and $1000 \Delta t$, respectively. (g-i) Overlapped isosurfaces $\phi=0$ for each column at $t=0,600 \Delta t$, and $1000 \Delta t$, respectively. Note that the axes are limited to show only $(-0.6,0.6)^{3}$. 


\section{Conclusions}

We presented two benchmark problems for the numerical discretization of the $\mathrm{CH}$ equation with a source term. The first benchmark problem is the growth of a disk or a sphere. If the source term is isotropic, then the growth should be isotropic. The second benchmark problem is the growth of a rotated ellipse or a rotated ellipsoid. Ideally, the growth should be independent of rotation. Therefore, it is essential to satisfy these two benchmark problems if a numerical discretization is reliable and accurate.

\section{Data Availability}

The data used to support the findings of this study are available from the corresponding author upon request.

\section{Conflicts of Interest}

The authors declare that they have no conflicts of interest.

\section{Acknowledgments}

Junseok Kim was supported by the National Research Foundation (NRF), Korea, under project BK21 FOUR.

\section{References}

[1] D. Anders and K. Weinberg, "Numerical simulation of diffusion induced phase separation and coarsening in binary alloys," Computational Materials Science, vol. 50, no. 4, pp. 1359-1364, 2011.

[2] J. W. Cahn and J. E. Hilliard, "Free energy of a nonuniform system. I. Interfacial free energy," The Journal of Chemical Physics, vol. 28, no. 2, pp. 258-267, 1958.

[3] F. Guillén-González and G. Tierra, "Unconditionally energy stable numerical schemes for phase-field vesicle membrane model," Journal of Computational Physics, vol. 354, pp. 67-85, 2018.

[4] B. S. Hosseini, S. Turek, M. Möller, and C. Palmes, "Isogeometric Analysis of the Navier-Stokes-Cahn-Hilliard equations with application to incompressible two-phase flows," Journal of Computational Physics, vol. 348, pp. 171-194, 2017.

[5] Y. Wang, C. Shu, H. B. Huang, and C. J. Teo, "Multiphase lattice Boltzmann flux solver for incompressible multiphase flows with large density ratio," Journal of Computational Physics, vol. 280, pp. 404-423, 2015.

[6] A. L. Bertozzi, S. Esedoglu, and A. Gillette, "Inpainting of binary images using the Cahn-Hilliard equation," IEEE Transactions on Image Processing, vol. 16, no. 1, pp. 285-291, 2007.

[7] X. Hu, Y. Li, and H. Ji, "A nodal finite element approximation of a phase field model for shape and topology optimization," Applied Mathematics and Computation, vol. 339, pp. 675-684, 2018.

[8] C. Liu, F. Frank, and B. M. Rivière, "Numerical error analysis for nonsymmetric interior penalty discontinuous Galerkin method of Cahn-Hilliard equation," Numerical Methods for Partial Differential Equations, vol. 35, no. 4, pp. 1509-1537, 2019.

[9] Z. Mu, Y. Gong, W. Cai, and Y. Wang, "Efficient local energy dissipation preserving algorithms for the Cahn-Hilliard equation," Journal of Computational Physics, vol. 374, pp. 654-667, 2018.

[10] J. M. Park, "Mathematical modeling and computational simulation of phase separation in ternary mixtures," Applied Mathematics and Computation, vol. 330, pp. 11-22, 2018.

[11] J. Zhu, L.-Q. Chen, and J. Shen, "Morphological evolution during phase separation and coarsening with strong inhomogeneous elasticity," Modelling and Simulation in Materials Science and Engineering, vol. 9, no. 6, pp. 499-511, 2001.

[12] A. C. Aristotelous, O. A. Karakashian, and S. M. Wise, "Adaptive, second-order in time, primitive-variable discontinuous Galerkin schemes for a Cahn-Hilliard equation with a mass source," IMA Journal of Numerical Analysis, vol. 35, no. 3, pp. 167-1198, 2015.

[13] A. Hawkins-Daarud, K. G. van der Zee, and J. Tinsley Oden, "Numerical simulation of a thermodynamically consistent four-species tumor growth model," International Journal for Numerical Methods in Biomedical Engineering, vol. 28, no. 1, pp. 3-24, 2012.

[14] V. Mohammadi and M. Dehghan, "Simulation of the phase field Cahn-Hilliard and tumor growth models via a numerical scheme: element-free Galerkin method," Computer Methods in Applied Mechanics and Engineering, vol. 345, pp. 919-950, 2019.

[15] J. Ren, D. Shi, and S. Vong, "High accuracy error estimates of a Galerkin finite element method for nonlinear time fractional diffusion equation," Numerical Methods for Partial Differential Equations, vol. 36, no. 2, pp. 284-301, 2020.

[16] H. Fakih, "A Cahn-Hilliard equation with a proliferation term for biological and chemical applications," Asymptotic Analysis, vol. 94, no. 1-2, pp. 71-104, 2015.

[17] H. Fakih, "Asymptotic behavior of a generalized Cahn-Hilliard equation with a mass source," Applicable Analysis, vol. 96, no. 2, pp. 324-348, 2017.

[18] E. Khain and L. M. Sander, "Generalized Cahn-Hilliard equation for biological applications," Physical Review E.vol. 77, no. 5, Article ID 051129, 2008.

[19] J. J. Benito, A. García, L. Gavete, M. Negreanu, F. Ureña, and A. M. Vargas, "On the convergence of the generalized finite difference method for solving a chemotaxis system with no chemical diffusion," Computational Particle Mechanics, vol. 8, no. 3, pp. 625-636, 2021.

[20] J. J. Benito, Á. García, M. L. Gavete, M. Negreanu, F. Ureña, and A. M. Vargas, "Convergence and numerical solution of a model for tumor growth," Mathematics, vol. 9, no. 12, p. 1355, 2021.

[21] M. I. P. Hidayat, "Meshless finite difference method with B-splines for numerical solution of coupled advection-diffusion-reaction problems," International Journal of Thermal Sciences, vol. 165, Article ID 106933, 2021.

[22] V. Mohammadi, M. Dehghan, and S. De Marchi, "Numerical simulation of a prostate tumor growth model by the RBF-FD scheme and a semi-implicit time discretization," Journal of Computational and Applied Mathematics, vol. 388, Article ID 113314, 2021.

[23] M. Smoka, M. Woniak, and R. Schaefer, "A three-level linearized time integration scheme for tumor simulations with Cahn-Hilliard equations," in International Conference on Computational Science, pp. 173-185, Springer, Cham, Switzerland, 2021.

[24] A. Kumar, "Isotropic finite-differences," Journal of Computational Physics, vol. 201, no. 1, pp. 109-118, 2004.

[25] M. Patra and M. Karttunen, "Stencils with isotropic discretization error for differential operators," Numerical 
Methods for Partial Differential Equations, vol. 22, no. 4, pp. 936-953, 2006.

[26] H. Assadi, "A phase-field model for non-equilibrium solidification of intermetallics," Acta Materialia, vol. 55, no. 15, pp. 5225-5235, 2007.

[27] D. J. Eyre, "An unconditionally stable one-step scheme for gradient systems," 1997.

[28] A. C. Aristotelous, O. Karakashian, O. Karakashian, and S. M. Wise, "A mixed discontinuous Galerkin, convex splitting scheme for a modified Cahn-Hilliard equation and an efficient nonlinear multigrid solver," Discrete \& Continuous Dynamical Systems-B, vol. 18, no. 9, pp. 2211-2238, 2013.

[29] W. L. Briggs and S. F. McCormick, A Multigrid Tutorial, SIAM, Philadelphia, PA, USA, 2nd edition, 2000.

[30] U. Trottenberg, A. Schüller, and C. Oosterlee, Multigrid, Academic Press, New York, NY, USA, 1st edition, 2000. 